\title{
Proper identification of RR Lyrae stars brighter than 12.5 mag ${ }^{\star}$
}

\author{
G. Maintz
}

Sternwarte der Universität Bonn, Auf dem Hügel 71, 53121 Bonn, Germany

e-mail: gmaintz@astro.uni-bonn.de

Received 12 April 2005 / Accepted 13 July 2005

\section{ABSTRACT}

RR Lyrae stars are of great importance for investigations of Galactic structure. However, a complete compendium of all RR-Lyraes in the solar neighbourhood with accurate classifications and coordinates does not exist to this day. Here we present a catalogue of 561 local RR-Lyrae stars ( $V_{\max } \leq 12.5 \mathrm{mag}$ ) according to the magnitudes given in the Combined General Catalogue of Variable Stars (GCVS) and 16 fainter ones. The Tycho 2 catalogue contains $\simeq 100$ RR Lyr stars. However, many objects have inaccurate coordinates in the GCVS, the primary source of variable star information, so that a reliable cross-identification is difficult. We identified RR Lyrae from both catalogues based on an intensive literature search. In dubious cases we carried out photometry of fields to identify the variable. Mennessier \& Colome (2002, A\&A, 390, 173) have published a paper with Tyc2-GCVS identifications, but we found that many of their identifications are wrong.

Key words. astrometry - stars: variables: RR Lyr - catalogs

\section{Introduction}

RR Lyrae stars (RR Lyrs) are classical variables of the instability strip. They are one of the primary distance indicators, which is why they have often been investigated to determine their absolute magnitude and their distances (see, e.g., references in Fernley et al. 1998). In addition, RR Lyrs provide an excellent sample of stars to investigate kinematics in the galaxy (see, e.g., Beers et al. 2000; Layden 1994; Layden et al. 1996; Dambis \& Rastorguev 2001; Martin \& Morrison 1998; Altmann \& de Boer 2000).

Several studies have recently been carried out on other horizontal-branch (HB) stars. Altmann et al. (2004) published a study of the orbits and halo distribution of sdB stars, extending earlier work by de Boer et al. (1997). A similar study on RHB stars has been completed, too (Kaempf et al. 2005). It thus appeared worthwhile to also investigate the orbits and the ensuing z-distribution of RR Lyrs (Maintz \& de Boer 2005).

For such purposes the distance of the stars as well as accurate data of proper motion and radial velocity are required. A good source for astrometric data is the Tycho-2 catalogue (Høg et al. 2000, Tyc2) since it provides astrometric data for more than 2.5 million stars. However, one first has to identify the RR Lyrs in that catalogue.

We took the Combined General Catalogue of Variable Stars (Kholopov et al. 1998, GCVS) as a basic source for compiling an unbiased sample of RR Lyrs. The GCVS is, as the authors

* Full Table 1 is only available in electronic form at the CDS via anonymous ftp to cdsarc.u-strasbg.fr $(130.79 .128 .5)$ or via http://cdsweb.u-strasbg.fr/cgi-bin/qcat?]/A+A/442/381 write, "the only reference source on all known variable stars ... The total number of designated variable stars has now reached 31918". However, the coordinates given in GCVS are not as accurate as one would like for, specifically, cross-correlations with other catalogues. The positions in GCVS are said to be accurate "to $1 \mathrm{~s}$ of arc". But we found that this accuracy is too optimistic. In some cases the coordinates denoted not the variable but another star. In even more cases, at the location given by the coordinates there was no star at all. According to the authors of GCVS, "the Sternberg Institute has started preparation of an electronic release GCVS 4.2 which will contain improved light elements, classifications etc., along with sufficiently accurate positional information".

In this paper we present a list of RR Lyrs, with $V_{\max } \leq$ $12.5 \mathrm{mag}$, all present in the GCVS, but now with absolutely reliable positions. It includes 286 Hipparcos and Tycho stars (ESA 1997; HIP), of which 104 only in Tyc2, as well as 273 in the GSC (Lasker et al. 1996), and a few USNO-A2 stars (the USNO-A2 is published by Monet et al. 1998). Our list contains the names used in each of these catalogues.

\section{Comparing catalogues}

As a first step we began with an identification of RR Lyr stars in the GCVS with a brightness at maximum of $V_{\max } \leq 12.5$ mag. We then proceeded to inspect their positions by looking up the original finding charts. It emerged from that already that several RR Lyrs were not identified properly. Thus a full inspection of all the basic data was required. 


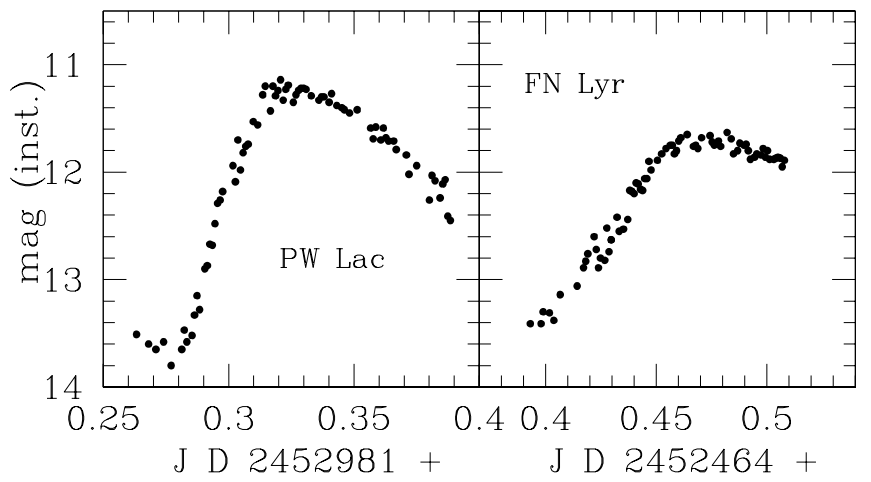

Fig. 1. Light-curve of PW Lac (left panel) and FN Lyr (right panel) observed at the observatory of Maintz. Such data was used to identify the RR Lyrae stars in their fields.

Because it was impossible to observe all RR Lyrs ourselves, and especially stars from the southern hemisphere could not be observed by us, we made a literature search to find the missing ones. We followed very strict principles. First, papers which give a finding chart or image identifying the variable by observing its light-curve were regarded as a reliable reference. Second, we used papers giving the Tyc2 or GSC number of the star, by the same criteria. Beside this we tried to find the original record of discovery and the finding chart given there. It takes considerable effort to do so, especially if only very old finding notes exist, which are not available electronically.

All images, finding charts and records of the discoverer were examined in the same way as our own observations. We compared them with the DSS and overlaid them with Tyc2 and GSC from the VizieR catalogue service using the Aladin interactive sky atlas. However, for some GCVS stars the identification was still elusive and it was decided to go and observe the fields anew to properly identify the particular RR Lyrs.

\section{Observation and identification of RR Lyrae stars}

The best way to identify RR Lyrs in the field of stars is to observe the fields and look for the star with RR Lyr like variability.

For 34 fields time series were obtained in Bonn, using a $8^{\prime \prime}$ Schmidt-cassegrain Meade telescope and an OES AlphaMini CCD-camera with an IRR-UV-Cut filter (B\&W 486). This equipment is fully sufficient for taking series of images for several hours allowing to define the light-curve. Usually about every minute an image was taken. For an example of light-curves see Fig. 1.

The data reduction was made with the OES-Fleischmann software provided with the camera. We identified the RR Lyr easily by its characteristic variability. We then compared our image with the DSS as described in Sect. 2. With this procedure we extracted name and coordinates of the star in question from the Tyc2 and GSC catalogue, respectively.

For 22 stars time series were observed at the Observatory Hoher List with the $1 \mathrm{~m}$ Cassegrain-Telescope using the Cassegrain focus with focal reducer $(f=368 \mathrm{~cm})$ and the HoLiCam $2048 \times 2048$ pix CCD camera. For the reduction of these CCD-images we used standard CCD reduction routines

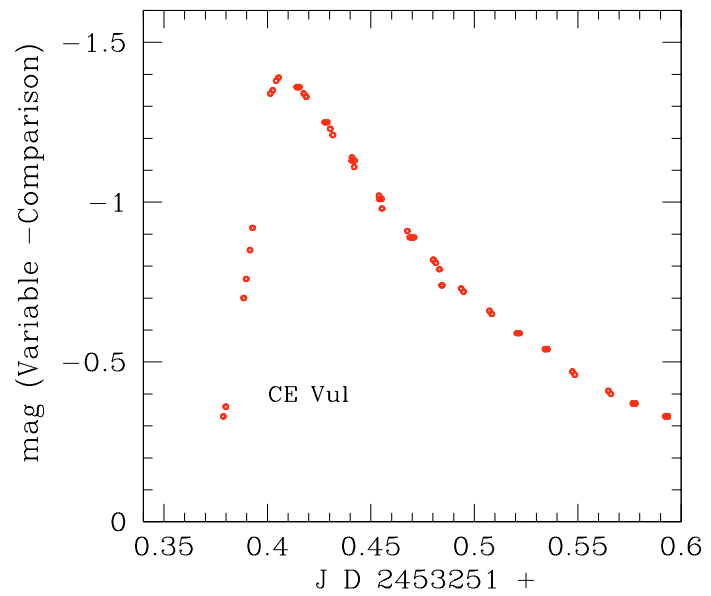

Fig. 2. Light-curve of CE Vul observed at the observatory "Hoher List" of the Sternwarte of the Univ. of Bonn. Such data was required to identify RR Lyrae stars in their fields.

(DAOPHOT under IRAF) to determine positions and magnitudes of all stars on the frames. The RR Lyr in question was identified by its light-curve (for an example see Fig. 2) and its identification number and coordinates were obtained by comparing with the DSS overlaying with the Tyc2 or GSC, too.

\section{The final catalogue}

We give all RR Lyrs with $V_{\max } \leq 12.5 \mathrm{mag}$ (according to GCVS) in one list, but we mention that the 186 RR Lyrs from the HIP which are included in our catalogue, are taken from HIP directly needing no further identification.

Our final catalogue contains 577 RR Lyrs with the following information in the columns

1: $\quad$ name of the variable (GCVS);

2+3: catalogue $(H=$ Hip, $T=$ Tyc2, $G=$ GSC, UA2 = USNO - A2) + number; an asterisk points at an individual note to the star ${ }^{1}$;

4+5: $\alpha, \delta(2000)$ given in the particular catalogue;

6: $\quad V$ magnitude in maximum light;

7: $\quad$ class of RR Lyr based on light-curve;

8+9: first epoch and period;

10: date of last observed maximum light known by us;

11: source of first epoch and period if not taken from GCVS or HIP.

If the star is taken from HIP, the magnitude in maximum light, type of variability, first epoch and period are from the HIP Variability Annex. If this information comes from another catalogue, it is from the GCVS, except when new information for first epoch and period are available in the literature.

The date of the last observation of maximum light mostly comes from amateur observers. They regularly observe variables of all kinds and they obtain light-curves of RR Lyrs as well, achieving correct maximum epochs. This column is, although not complete, very useful to make predictions of maximum light.

\footnotetext{
1 The source of identification is available from gmaintz@astro.uni-bonn.de
} 
Table 1. 561 RR-Lyrae Stars with maximum light $\leq 12.5$ mag according to GCVS plus 16 fainter ones.

\begin{tabular}{|c|c|c|c|c|c|c|c|c|c|c|}
\hline $\begin{array}{l}\text { Name } \\
\text { of star }\end{array}$ & Cat & $\mathrm{Nr}$ & $\alpha_{2000}$ & $\delta_{2000}$ & $\begin{array}{r}V_{\max } \\
(\mathrm{mag})\end{array}$ & Type & $\begin{array}{l}\text { First ep. } \\
\text { (JD) }\end{array}$ & $\begin{array}{l}\text { Per. } \\
\text { (d) }\end{array}$ & $\begin{array}{l}\text { Last observ. } \\
\text { (JD) }\end{array}$ & $\begin{array}{l}\text { Source } \\
\text { of period }\end{array}$ \\
\hline SW And & $\overline{\mathrm{H}}$ & 1878 & 002343.090 & 292403.62 & 9.22 & RRab & 2448500.0384 & 0.4422620000 & 53260.1300 & \\
\hline XX And & $\mathrm{H}$ & 6029 & 011727.415 & 385702.03 & 10.20 & RRab & 2448500.6580 & 0.7227550000 & 53045.3410 & \\
\hline AT And & $\mathrm{H}$ & 116958 & 234230.832 & 430051.66 & 10.51 & RRab & 2448500.2310 & 0.6169170000 & 53232.5870 & \\
\hline BK And & $\mathrm{G}$ & 323500127 & 233506.022 & 410610.51 & 12.50 & RRab & 2429146.4500 & 0.4215985000 & 51321.8076 & \\
\hline CI And & $\mathrm{H}$ & 8939 & 015508.294 & 434556.47 & 11.78 & RRab & 2448500.0022 & 0.4847280000 & 52695.3060 & \\
\hline DK And & $\mathrm{G}^{*}$ & 364500701 & 232845.910 & 503429.35 & 12.50 & RR: & 2429130.4070 & 0.2436553000 & & \\
\hline DM And & $\mathrm{T}$ & 277300253 & 233200.707 & 351148.87 & 12.40 & RRab & 2435717.4310 & 0.6303890000 & 51335.8300 & \\
\hline DR And & $\mathrm{T}$ & 228600352 & 010510.707 & 341306.24 & 12.00 & RRab & 2437220.3190 & 0.5631180000 & 52620.4385 & \\
\hline DU And & $\mathrm{G}$ & 283600362 & 023031.337 & 405033.11 & 12.50 & $\mathrm{RR}$ & 2436051.4500 & 0.6067160000 & 51469.4641 & \\
\hline OV And & $\mathrm{T}$ & 278701874 & 002044.206 & 404940.80 & 10.40 & RRab & 2439026.4780 & 0.4705810000 & 52983.4265 & 1 \\
\hline V395 And & $\mathrm{H}$ & 117111 & 234432.143 & 462248.59 & 7.57 & RRc: & 2448500.2660 & 0.3423280000 & & \\
\hline WY Ant & $\mathrm{H}$ & 50289 & 101604.946 & -294342.41 & 10.40 & RRab & 2448500.5339 & 0.5743410000 & 51869.6630 & \\
\hline SY Aps & $\mathrm{G}^{*}$ & 926500960 & 143924.130 & -724935.62 & 12.20 & RR: & 2451904.0650 & 0.2789100000 & & 2 \\
\hline TY Aps & $\mathrm{H}$ & 72444 & 144850.012 & -711941.88 & 11.27 & RRab & 2448500.4217 & 0.5016950000 & 52823.5250 & \\
\hline TY Aps & $\mathrm{H}$ & 72444 & 144850.012 & -711941.88 & 11.27 & RRab & 2448500.4217 & 0.5016950000 & 52823.5250 & \\
\hline UY Aps & UA2 & 015014253525 & 145934.440 & -714753.77 & 12.00 & RRab & 2425326.5400 & 0.4825200000 & 51921.9800 & \\
\hline VX Aps & $\mathrm{G}$ & 942900076 & 155956.690 & -751321.00 & 11.50 & RRab & 2434239.3610 & 0.4845780000 & 51927.5010 & \\
\hline$\ldots$ & $\cdots$ & $\cdots$ & $\cdots$ & $\cdots$ & $\cdots$ & $\ldots$ & $\cdots$ & $\ldots$ & $\cdots$ & \\
\hline$\cdots$ & $\cdots$ & .. & $\cdots$ & $\ldots$ & $\cdots$ & $\ldots$ & $\cdots$ & $\cdots$ & $\cdots$ & \\
\hline
\end{tabular}

The sources of first epoch and period if not from HIP or GCVS:

1 Rossiger, S., \& Busch, H. 1988, Mitt. Verand. Sterne 11, 133.

2 A. Paschke, priv. communication.

...

$\cdots \cdots$

Individual notes:

DK And type EW is also possible. A. Paschke, priv. communication.

SY Aps type EW is also possible. A. Paschke, priv. communication.

.. $\quad \ldots \quad \ldots$

.. $\quad \cdots$

If a star could not be identified in its field, because no second source for identification besides the GCVS was available (and no variable was found in our observation of the field) our catalogue has 999999999 as number. This happened in eight cases. The position given for these stars is taken from the GCVS. If there are no data for period or first epoch in the GCVS and no information is found otherwise, its first epoch and period is given as 9999999.9999 and 9.99999999, respectively.

For 6 stars without Tyc2 or GSC number we give identifications and coordinates (2000) from the USNO-A2.0. Magnitudes and elements are taken from the GCVS.

For three stars (DM And, CQ Lac and UZ Eri) being members of Tyc2, there are no proper motions available in the Tyc2. Therefore those stars are not useful for further investigations based on Tyc2.

$\mathrm{XCMi}$ is identified as GSC 168-406 while Schmidt et al. (1995) identified it as GSC 168-562. However, comparing their finding chart with the chart from Tsesevisch \& Kazanasmas (1971), we found that in both charts there is only one star at the position of $\mathrm{X} \mathrm{CMi}$ while the POSS gives a close pair. GSC 168-562 is the preceding star of the pair and is very faint
(15.25 mag according to GSC III aJ). That is why we adopt that X CMi is the following star (168-406) with a magnitude of 12.34 mag (GSC III aJ).

Mennessier \& Colome (2002, hereafter MC) gave results for 172 RR Lyrae stars identified to be Tyc2 stars. They did the identification blindly, using the GCVS as source of the positions and magnitudes of the RR Lyrs. They were successful in finding many RR Lyrs at Tyc 2 star positions. But they did not find all and, as it turned out during our search, their list is wanting since they misidentified a large fraction of their stars.

For 78 stars of their 172 we agree with the identifications of MC. In 93 cases we found their stars being misidentified. We found 27 Tyc2 stars which are not given by MC. From the misidentified ones we found 84 in the GSC and 3 in the USNO-A2, while for 3 Tyc2 stars (NOCas, V672 Aql, $\mathrm{V} 1823 \mathrm{Cyg}^{2}$ ) we found another identification number than given by MC. The misidentification mostly happens, when the star was fainter than the magnitudes given in the GCVS. Of the MC paper stars, 3 are not mentioned in our list: V1823 Cyg (see IBVS 4997), UU Cam (Schmidt et al. 1995), CE Aqr

2 According to IBVS Nr 4997, V1823 Cyg is a W-UMa star and not a RR Lyrae star. It is of course not included in our catalogue. 
(A. Paschke, priv. commun.), because they are not RR Lyrs. 14 stars of their list are fainter than our limiting magnitude, but we added these stars to our list for completeness.

\section{Summary}

We present a catalogue listing 561 RR Lyrs with $V_{\max } \leq$ 12.5 mag (plus 16 fainter ones) with exact coordinates. Most of them come from the GCVS. 21 have recently been recovered (of the new found RR Lyrs 2 have a $V_{\max }$ of 12.52 and 12.53 mag respectively). 27 RR Lyrs are previously unknown as members of Tyc2. Data of first epoch and period are given if available. The dates of the last maxima observed come mostly from amateur observers and (even if this data is incomplete) allow to calculate trust-able predictions.

As an example, the beginning of the catalogue is given in Table 1.

Acknowledgements. This research has made use of the Simbad database, operated at CDS, Strasbourg, France. We thank Karl Wälke and Wolfgang Grimm who donated the Atlas of Finding Charts for Variable Stars by Tsesevisch and Kazanasmas. We thank Franz Agerer, Wolfgang Moschner, Konstantin von Poschinger and K. Bernhard for sending several identifications of stars from their observations. We thank Anton Paschke for sending maximum light times of recent observations.

\section{References}

Altmann, M., \& de Boer, K. S. 2000, A\&A, 353, 135

Altmann, M., Edelmann, H., \& de Boer, K. S. 2004, A\&A, 414, 181

Beers, T. C., Chiba, M., Joshii, Y., et al. 2000, AJ, 119, 2688

de Boer, K. S., Aguilar Sanchez, Y., Altmann, M., et al. 1997, A\&A, 327, 577

Dambis, A. K., \& Rastorguev, A. S. 2001, AstL, 27, 108

ESA, 1997, The Hipparcos and Tycho Catalogues, ESA SP1200, and its Variability Annex: Periodic variables

Fernley, J., Barnes, T. G., Skillen, I., et al. 1998, A\&A, 330, 515

Høg, E., Fabricius, C., Makarov, V. V., et al. 2000, Tycho-2 catalogue (Tyc2)

Kaempf, K. S., de Boer, K. S, \& Altmann, M. 2005, A\&A, 432, 879

Kholopov, P. N., Samus, N. N., Durlevich, O. V., et al. 1990, General Catalogue of Variable Stars, 4rd edn., Vol. IV (Moscow: Nauka) (GCVS)

Lasker, B. M., Russel, J. N., \& Jenkner, H. 1996, The HST Guide Star Catalogue, Version 1.2

Layden, A. C. 1994, AJ, 108, 1016

Layden, A. C., Hanson, R. B., Hawley, S. L., et al. 1996, AJ, 112, 2110

Maintz, G., \& de Boer, K. S. 2005, A\&A, 442, 229

Martin, J. C., \& Morrison, H. L. 1998, AJ, 116, 1724

Mennessier, M. O., \& Colomé, J. 2002, A\&A, 390, 173

Schmidt, E. G., Chab, J. R., \& Reiswig, D. E. 1995, AJ, 109, 1239

Monet, D., Bird, A., Canzian, B., et al. 1998, A Catalogue of Astrometric Standards (USNO-A V2.0)

Tsesevisch, V. P., \& Kazanasmas, M. S. 1971, Atlas of Finding Charts for Variable Stars, Moscow 